\title{
Age and baseline values predict 12 and 24-month functional changes in type 2 SMA
}

Giorgia Coratti ${ }^{1,2^{\circ}}$, Maria C Pera ${ }^{1,2^{\circ}}$, Simona Lucibello ${ }^{1,2^{\circ}}$, Jacqueline Montes ${ }^{3,4}$, Amy Pasternak $^{5}$, Anna Mayhew $^{6}$, Allan M Glanzman ${ }^{7}$, Sally Dunaway Young ${ }^{8}$, Marika Pane $^{2}$, Mariacristina Scoto ${ }^{9}$, Sonia Messina ${ }^{10}$, Nathalie Goemans ${ }^{11}$, Andres Nascimiento Osorio ${ }^{12}$, Francesco Muntoni ${ }^{16}$, Darryl C. De Vivo ${ }^{3}$, Richard Finkel $^{11 *}$, Eugenio Mercuri ${ }^{1,2 \# *}$ on behalf of the ISMAC group.

${ }^{\circ}$ all co-first authors

* both co-last authors

${ }^{1}$ Pediatric Neurology, Università Cattolica del Sacro Cuore, Rome, Italy

${ }^{2}$ Centro Clinico Nemo, Fondazione Policlinico Universitario Agostino Gemelli IRCCS, Rome, Italy

${ }^{3}$ Departments of Neurology and Pediatrics, Columbia University Irving Medical Center, New York, USA

${ }^{4}$ Departments of Rehabilitation and Regenerative Medicine and Neurology, Columbia University Irving Medical Center, New York, USA

${ }^{5}$ Departments of Neurology, Boston Children's Hospital, Harvard Medical School, Boston, MA

${ }^{6}$ Neuromuscular Unit, Neuropaediatrics Department, Institut de Recerca Hospital Universitari Sant Joan de Deu, Barcelona, Spain.

${ }^{7}$ The John Walton Muscular Dystrophy Research Centre,Newcastle University, Integrated Laboratory Medicine Directorate, Institute of Genetic Medicine, Newcastle Upon Tyne NHS Foundation Trust, Newcastle Upon Tyne, UK

${ }^{8}$ Department of Physical Therapy, The Children's Hospital of Philadelphia, Philadelphia ${ }^{9}$ Department of Neurology, Stanford University, Stanford, California, USA.

${ }^{10}$ Dubowitz Neuromuscular Centre, UCL Institute of Child Health \& Great Ormond Street Hospital, London

${ }^{11}$ Nemours Children's Hospital, University of Central Florida College of Medicine, Orlando, USA

${ }^{21}$ Department of Clinical and Experimental Medicine and Centro Clinico Nemo Sud, University of Messina, Messina, Italy 
${ }^{32}$ Unit of Neuromuscular and Neurodegenerative Disorders, Department of Neurosciences, IRCCS Bambino Gesù Children's Hospital, Rome, Italy

${ }^{14}$ Department of Child Neurology, University Hospitals Leuven, Leuven, Belgium

${ }^{15}$ Neurorehabilitation Unit, University of Milan, The NEMO Clinical Center in Milan, Italy

${ }^{16}$ NIHR Great Ormond Street Hospital Biomedical Research Centre, London, United Kingdom

Corresponding author:

Eugenio Mercuri

Pediatric Neurology, Catholic University

Largo Gemelli 8, 00168 Rome, Italy

Tel.: +390630155340; fax: +390630154363; E-mail: eugeniomaria.mercuri@unicatt.it

Authors and ISMAC group declaration of interest:

GC, RDS, JM, ESM, AM, AMG, SDY, RS, MP, SM, ADA, EA, BTD, EB, VAS, JD, FM, DCDV, RF, EM reports personal fees as consultant, PI or member on advisory board form BIOGEN S.R.L. outside the submitted work,

$G C, M C P, J M, E S M, A M, A M G, S D Y, M S, B T D, E B, J D, F M, F B, D C D V, R F, E M$ reports personal fees consultant, $\mathrm{PI}$ or member on advisory board from ROCHE,

GC reports personal fees GENESIS PHARMA and Biologix as consultant outside the submitted work, $G C, R D S, E S M, A M, A M G, R S, M P, S M, B T D, E B, V A S, J D, F M, D C D V, R F, E M$ reports personal fees consultant, PI or member on advisory board form AVEXIS outside the submitted work, $A P, S D Y, R S$ reports personal fees from as grant SMA FOUNDATION outside the submitted work, ESM, SDY, EM report personal fees consultant, PI or member on advisory board from SCHOLAR ROCK outside the submitted work, MS reports personal fees as CO-PI from SMA REACH UK outside the submitted work, $A D A, J D, R F$ reports personal fee as consultant, $\mathrm{PI}$ or member on advisory board from NOVARTIS outside the submitted work, 
SL, JEE, SC, EM, LA, AF, MVDH, FS, CMB, MC, RML, MM, MS, GV, TD, NG, ANO, have nothing to disclose. All authors from Italy, UK and US are part of institutions that receive funding for a SMA disease registry (ISMAR) 


\section{Abstract}

The aim of this retrospective study was to establish the range of functional changes at 12 and 24month in 267 type 2 Spinal Muscular Atrophy (SMA) patients with multiple assessments. We included 652 Hammersmith Functional Motor Scale Expanded (HFMSE) assessments at 12 monthand 305 at 24 month- intervals. The cohort was subdivided by functional level, Survival of Motor Neuron copy number and age.

Stable scores ( \pm 2 points) were found in $68.25 \%$ at 12 months and in $55.41 \%$ at 24 months. A decrease $\geq 2$ points was found in $21 \%$ at 12 months and in $35 \%$ at 24 months. An increase $\geq 2$ points was found in $11 \%$ at 12 months and $9.5 \%$ at 24 months.

The risk of losing $\geq 2$ points increased with age and HFMSE score at baseline both at 12 and 24month. For each additional HFMSE point at baseline, the relative risk of a $>2$ point decline at 12 months increases by $5 \%$ before age 5 years $(p=0.023)$, by $8 \%$ between 5 and $13(p<0.001)$ and by $26 \%$ after 13 years $(p=0.003)$.

The combination of age and HFMSE scores at baseline increased the ability to predict progression in type 2 SMA.

Keywords: Spinal Muscular Atrophy, Outcome measures, Hammersmith Functional Motor Scale Expanded, Neuromuscular disorders 


\section{Introduction}

Spinal muscular atrophy (SMA) is an autosomal recessive neuromuscular disorder caused by mutations in survival motor neuron 1 gene (SMN1). Patients affected by the intermediate form (type 2) typically acquire the ability to sit independently but do not achieve independent walking. Type 2 patients may show relatively small changes over 12 months [1], but some decline can be observed when two or three years are considered especially in the older patient population [2]. The recent availability of new treatments is likely to modify the known natural history of SMA [3]. The results of the pivotal study CHERISH (NCT02292537) using Nusinersen in later onset SMA [4], showed a mean increase of 4 points on the Hammersmith Functional Motor Scale Expanded (HFMSE) after 15 months of treatment, as opposed to the minimal negative changes observed in the placebo group. The study, however, only included patients with an age at screening between 2 and 12 years, able to sit independently, with HFMSE scores between 10 and 54 at screening and in the absence of severe scoliosis or contractures, respiratory insufficiency or gastric tube for nutrition.

The commercial availability of Nusinersen to all the SMA patients in several countries $[5,6]$ is allowing to establish the possible effect of the drug also in patients with broader clinical severity compared to those recruited in the CHERISH study, including older and more severely affected type 2 children and adults. One of the difficulties with the interpretation of the real-world data is that, being collected as part of clinical practice, there is no control group and therefore has to be interpreted in relation to previously collected natural history data. The interpretation is difficult due to the increasing realization that the course of progression of SMA type 2 is variable in patients of different age and severity $[7,8]$. Some spontaneous improvements may occur in untreated type 2 young patients while after the age of 5 and until 13-14 years there is often a 
sharp decline with a marked loss of HFMSE scores [8-10]. Another study has identified the percentage of patients who would remain stable (within +2 points) on the HFMSE or would have positive or negative changes more than 2 points in different age groups [7]. Although the patients in the previous study [7] represent the largest cohort with longitudinal functional data reported so far in type 2 SMA, the number of patients within each category was relatively small when they were subdivided according to type, age, baseline values or SMN2 copy number. The possibility to collect additional data from the previous participating centers and from new centers allowed us to obtain a much larger dataset, ask more specific questions and have a longer follow up. In this paper we focused on patients with type 2 SMA. The aim was to report the range of HFMSE changes at 12 and 24 months in 267 type 2 SMA patients and to establish percentage of stable scores versus the possibility of improving or showing a decline in relation to age, baseline levels and SMN copies.

\section{Materials and methods}

The study was performed by retrospectively collecting data from different international datasets, including the three networks that are part of the international SMA consortium (iSMAc) in the United States (the Pediatric Neuromuscular Clinical Research Network for SMA), in Italy and in the UK as part of SMA Reach. Data from two additional centers coordinating natural history studies in Spain and Belgium were also included.

All patients with a genetically confirmed diagnosis of SMA and a clinically confirmed diagnosis of type 2 SMA were considered for inclusion, therefore including all patients who had been seen in the neuromuscular clinics of the participating centers using the same criteria used in our previous collaborative study on patterns of progression [7]. Only patients with at least two assessments at 12-month interval were selected for this study. Patients in whom one of the two performances 
was reported by the examiners as not reliable, because affected by transient pain, fractures, recent pneumonia, or other infections, intercurrent surgery, or any other factor that affected temporarily one of the two assessments, were excluded from the analysis. Similarly, according to studies suggesting that the introduction of albuterol may cause a sudden improvement in scores that can persist for the first 12 months [11], we excluded those who had started treatment in the 12 months before the study or who started the treatment between the two assessments. Data from patients participating in clinical trials were also excluded.

As part of the activities of the participating networks all participants or their guardians provided written informed consent approved by the respective institutional review boards.

HFMSE

The scale consists of 33 items, investigating the child's ability to perform various activities $[12,13]$. Each activity (item) is scored on a 3-point scoring system, with a score of 2 for "performs without modification", 1 "performs with modification/ adaption" and 0 for "unable to perform". A total score can be achieved by summing the scores for all the individual items. The total score can range from 0 , if all the activities are failed, to 66 , if all the activities are achieved. All items have to be tested without spinal jacket or orthoses.

\section{Training sessions}

The same procedure manual was shared by all the participating networks. As part of the activity of each network, individual evaluators were trained at in-person meetings in US and in Europe. The evaluators who performed the assessments included in the present study were the same who participated in the reliability studies. As part of the studies both in Europe and US, evaluators have regular annual refresher trainings with scale item and scoring review. 
Statistical analysis

The HFMSE was evaluated longitudinally over a 12 and 24-month period of time. Each patient could contribute with multiple assessments at 12-month intervals. As for each interval the patient was at a different age and, based on the course of the disease, could also have a different HFMSE score, each interval was considered independently, with a new age and HFMSE baseline for each calculated interval.

Summary statistics ( $N$, mean, median, SD, range) were used to analyze age, HFMSE at baseline and 12 months, changes at HFMSE over 12 months. The cohort was further subdivided by functional level (sitters/non-sitters; HMFSE score $<10 / 11-22 />22$ ), by SMN2 copy number and by age (<5/5$13 />13)$. Ability to sit independently was defined as a score of 2 on item 1 of the HFMSE.

The cut off points on the HFMSE were chosen based on the decimal classification [12], with scores between 0 and 10 identifying patients with type 2.0 to type 2.2 , i.e the weakest end of the spectrum of type 2 with patients unable to perform full rolling. Scores between 11 and 22 identified patients with type 2.3 to 2.4 , i.e those generally able to roll and prop. Scores above 22 identified children at the best end of the spectrum ( 2.5 and above) who were able to perform four-point kneeling and other activities up to standing.

The cut off point for age were chosen based on our previous observation reporting slopes of progression in type 2 at different age points [8].

A multinomial logistic regression model was used to assess changes in the slope of baseline values of the HFMSE ( $<-2$ change; $-2,2$ change and $>2$ change) at different cut points of age $(<5$ years; between 5 and 13 years and after 13 years) including different variables, such as age (in years), HFMSE scores at each baseline interval and the SMN2 copy number. The stability of change $(-2,2$ 
change) represents the reference category and, in order to taking account, the intra-subject correlation, a random effect was included in the regression model.

The 12 and 24-month changes in the HFMSE scores were compared between age classes by an analysis of variance, adjusting for baseline. As previous studies have shown that the great majority of patient variability is within +/- 2 points, we considered three ranges of change in the HFMSE. The percentage of patients with a change $<-2$ (significant decline), between -2 and 2 (nonsignificant variation), and $>2$ points (significant improvement) was compared across age classes by a chi-square test (a multinomial model was used to adjust for baseline values).

\section{Results}

The cohort included 652 12-month assessments from 267 patients, 150 males and 117 females. An additional 21 assessment could not be included because the patients had intercurrent surgery $(n=11)$, fractures $(n=5)$, recent pneumonia $(n=3)$ or poor cooperation $(n=2)$.

The age of the patients ranged between 30 months and 34 years at baseline (mean 9.41, SD 6.32). One hundred and four of the 267 patients had already been part of previous natural history studies[2, 7].

Of the 652 12-month assessments included, 541 assessments were from sitters and 111 from nonsitters patients, i.e. type 2 patients who lost independent sitting.

At 24 months there were 305 assessments from 147 patients, 77 males and 70 females. Their age ranged between 30 months and 33.40 years at baseline (mean 8.70, SD 5.91). Two-hundred sixtyseven assessments were from sitters and 38 from non-sitters patients.

HFMSE

12-month changes 
The HFMSE scores ranged between 0 and 41 (mean 11.55, SD +9.24) (Fig 1).

The 12-month change ranged between -13 and +12 (mean -0.68, SD +3.28) (Fig 2A-2C).

Of the 652 assessments, 445 (68.25\%) had HFMSE changes +2 points, 135 (20.71\%) had a decrease of more than 2 points and $72(11.04 \%)$ had an increase of more than 2 points.

\section{4-month changes}

At 24 months, the HFMSE scores ranged between 0 and 36.00 (mean 12.32, SD +8.85).

The 24-month changes ranged between -15 and +15 (mean -1.74, SD +3.89) (Fig 2B-2D).

Of the 305 assessments, 169 (55.41\%) had HFMSE changes +2 points, 107 (35.08\%) had a decrease of more than 2 points and $29(9.50 \%)$ had an increase of more than 2 points.

HFMSE changes and SMN2 copy number

At 12 months, of the 652 assessments, 1 was from patients with 1 copy of SMN2 gene, 51 from patients with 2 copies of SMN2 gene, 490 from patients with 3 copies of SMN2 gene and 10 from patients with 4 copies of SMN2 gene. In 100 the SMN2 gene copy number was unknown. At 24 months, of the 305 assessments, 19 were from patients with 2 copies of SMN2 gene, 241 with 3 copies of SMN2 gene and 5 with 4 copies of SMN2 gene.

There was no correlation between SMN2 copy number and HFSME scores at baseline or with HFMSE changes over 12 months $(p=0.490)$.

\section{HFMSE changes and functional status}

\section{$\underline{\text { Non sitters }}$}

The HFMSE changes were between -2 and +2 (mean -0.02 , SD +0.77$)$ at 12 months and between 2 and +4 (mean $-0.05, S D+1.00)$ at 24 months.

Of the 111 12-month assessments, 110 (99.09\%) had HFMSE changes +2 points, none (0\%) had a decrease of more than 2 points and only $1(0.90 \%)$ had an increase of more than 2 points. 
Of the 38 24-month assessments, 37 (97.36\%) had HFMSE changes +2 points, no one (0\%) had a decrease in scores of more than 2 points and only 1 (2.56\%) had an increase in scores of more than 2 points.

\section{$\underline{\text { Sitters }}$}

The changes were between -13 and +12 (mean $-0.83, S D+3.56)$ at 12 months and between -15 and +15 (mean -1.99, SD +4.09) at 24 months.

Of the 541 12-month assessments, 335 (61.92\%) had HFMSE changes + or < 2 points, 135 (24.95\%) had a decrease of more than 2 points and 71 (13.12\%) had an increase of more than 2 points.

Of the 267 24-month assessments, 131 (49.25\%) had HFMSE changes + 2 points, 107 (40.03\%) had a decrease of more than 2 points and $28(10.53 \%)$ had an increase of more than 2 points.

HFMSE changes and age

Details of the patients included in the age subgroups are reported in table 1 and supplementary table 1. In the assessments performed before the age of 5 years, the 12 months changes $(n=183)$ ranged between -12 and +12 (mean: 0.40, SD: 4.09) and the 24-month changes ( $n=97)$ between 15 and +15 (mean: -0.51 , SD: 4.80$)$.

In the assessments performed in patients between 5 and 13 years the 12 -month change $(n=352)$ ranged between -13 and +12 (mean: -1.39 , SD: +3.08 ) and the 24 -month changes $(n=166)$ between -12 and +5 (mean: $-2.81, \mathrm{SD}:+3.36)$.

In the assessments performed in patients older than 13 years the 12 -month changes $(n=117)$ ranged between -10 and 3 (mean: $-0.26, S D:+1.37)$ and the $24-$ month changes $(n=42)$ between -5 and 4 (mean: -0.38, SD: +1.65) (Fig 3) 
Each age subgroup was further subdivided by HFMSE scores: $<10,11-22,>22$. Table 1 and supplementary table 1 shows details of the distribution of scores and of the possible changes at 12 and 24 months in both sitters and non-sitters. For the sitter subgroup further details on the frequency distribution of stable versus increase or decrease of more than 2 points is illustrated in figure 4.

Using multinomial regression, we found that all variables included in the regression model, except for SMN2 copies, were useful to explain both 12 and 24 months HFMSE changes.

The risk of losing more than two points after 12 months increases with age and HFMSE score at baseline. For each additional point at baseline, the relative risk increases $6.8 \%$ before 5 years $(p=$ $0.005), 9.7 \%$ between 5 and 13 years $(p<0.001), 18 \%$ after 13 years $(p=0.017)$.

The risk of gaining more than two points increased before the age of 5 but no variable was significantly predictive.

\section{Discussion}

The results of the present study confirm our and others recent findings that although the mean HFMSE changes over time may be limited, there is a large variability and a wide range of scores, especially when assessing a population of patient with wide age range variability [7]. In this paper we included only patients above the age of 30 months according to the previous HFMSE validation in young infants as, even if the scale can be often administered below this age, this would be associated with a higher risk of poor cooperation. The possibility to have a large cohort of type 2 patients allowed us to expand the previous findings. We initially focused on the 12-month changes, as we had a much larger number of assessments at 12 than at 24 months and this is also the timeframe mostly used in clinical trials. We also analyzed the 24-month data, although the 
cohort was relatively smaller, to observe if, as reported in recent natural history studies, the changes were more obvious in a longer time interval [1, 2].

Our results confirmed that the great majority of type 2 patients (68.25\%) remained stable or with minimal changes over 12 months. The possibility to maintain stable scores over 2 years was lower (55.41\%). In order to exclude a possible bias, we analyzed separately type 2 patients who had lost the ability to sit. In these patients, who were generally older than 13 years, the baseline HFMSE scores were, as expected, always lower than 4 and often lower than 2, with a mean score of 0.65 (+1.05). With the exception of one child, younger than 5 years, their HFMSE scores remained stable both at 12 and 24 months. As previously reported, in this age group the HFMSE should not be the primary functional measure due to the low scores already at baseline [14] and should be complemented with the assessment of upper limb function using functional scales such as the Revised Upper Limb Module (RULM) [15], that have been found to be more sensitive to detect functional changes for weak type 2 SMA [16]. Unfortunately, as the RULM was developed only in the last few years, it was not systematically used in all the patients in the present study. Even excluding the non-sitters, the percentage of type 2 patients with stable scores was still relatively high (61.92\% at 12 and $49.25 \%$ at 24 months) while the chances of an improvement was approximately $13 \%$ at 1 year and $10 \%$ at 2 years. As previously reported, the chances of improvements were much higher in the younger group, below age 5 years [7]. This group, however, was the most heterogeneous group in terms of progression. While we confirmed that children below the age of 5 years had a relatively high possibility of improving more than 2 points after 12 months, the improvement only occurred in $26.97 \%$, while $54.49 \%$ had stable scores and nearly $19 \%$ had negative changes. This observation is important as in clinical practice or in interventional studies improvements of more than 2 points in this age group are often dismissed 
as compatible with natural history while this observation is only true in less than $27 \%$ of the patients in this age range with a further reduction (21\%) at 24 months.

The chances of improvements at 12 months dropped to approximately $7 \%$ between the age of 5 and 13 years and to less than $2 \%$ in the older group. In the 5 to 13 years group there was a parallel increase (33\%) in the number of patients who had negative changes over 12 months that increased to over $50 \%$ at 24 months. The group assessed after 13 years had the highest percentage of stable scores at 12 months (93.85\%) and also at 24 months (83.33\%), while there was a relative increase of patients with negative changes (12.50\%).

When we looked at the possible effect of other variables, we found that age was not the only determinant as the changes also reflected the functional status (non sitters/sitters) and baseline values. The risk of losing more than two points increased with age and HFMSE score at baseline. More specifically, for each additional point at baseline, the relative risk of negative changes at 12 months increased by $5 \%$ before age 5 years $(p=0.023)$, by $8 \%$ between years 5 and $13(p<0.001)$, and $26 \%$ after age 13 years $(p=0.003)$. The opposite was also true as the possibility to improve on the scales was extremely low in patients with high scores. This was surprising, to some extent, as despite patients with the highest scores at baseline $(>22)$ are likely to have more preserved motoneurons activity, they not only did not show an increase in scores over time but, irrespective of the age, generally showed a sharper decline both at 12 and 24 months. These findings suggest that these patients may have reached the maximal functional capacity with their existing motorneurons and that, having achieved high scores, may have more points to lose, especially with the development of contractures, scoliosis and general growth.

SMN2 copy number was associated with different scores at baseline, with patients with 3 copies having overall lower scores than those with more than 3 , but did not have a significant effect on the magnitude of changes. The lack of correlation with SMN2 copy numbers is probably related to 
the fact that over nearly $90 \%$ of the patients had 3 copies and the groups with 1, 2 or more than 3 copies were poorly represented. Unfortunately, a number of the older patients who had a less recent diagnosis did not have SMN2 copy number available and many of them refused to give consent for additional tests.

In conclusion, our results confirm that although the mean changes were very small at $12(-0.68)$ and 24 months (-1.74), the individual changes varied widely. An increase of more than two points was relatively uncommon, while there was a higher possibility to show a decrease of more than 2 points at 12 months that further increased at 24 months.

The combination of age and HFMSE scores at baseline increased the possibility to predict the progression at 12 and 24 months. Subdividing patients according to both variables helped to narrow the variability of the responses, providing more detailed information on the possibility to remain stable or to have positive or negative changes in the different subgroups.

Our findings provide reference data for the individual subgroups that will be of help at the time of interpreting the results of cohorts of patients who are treated with the newly available drugs or undergo other types of intervention. This will hopefully also help clinicians, patients and families, at the time of deciding whether to start a treatment, on setting up the right expectations based on the patient's baseline characteristics. According to these findings the expectation on a possible response to treatment should therefore be interpreted differently according to the age and function of the patient. The possibility to have stable scores over 12 months or longer, has a different meaning if observed in younger patients or in those between the age of 5 and 14 years with high HFMSE scores, who are more likely to decline.

A more robust statistical approach using trajectory analysis, as recently used in Duchenne muscular dystrophies and other diseases $[17,18]$, may allow to use a larger number of variables, beyond age and baseline, to further predict more accurate trajectories of progression. 


\section{Acknowledgments:}

We are thankful to the Italian Telethon, the SMA Reach UK and SMA Foundation for contributing to fund this study.

Funding: This work was supported by the Italian Telethon, the SMA Reach UK and SMA Foundation. 


\section{References}

[1] Kaufmann P, McDermott MP, Darras BT, Finkel R, Kang P, Oskoui M, et al. Observational study of spinal muscular atrophy type 2 and 3: functional outcomes over 1 year. Arch. Neurol. 2011;68:779-86.

[2] Kaufmann P, McDermott MP, Darras BT, Finkel RS, Sproule DM, Kang PB, et al. Prospective cohort study of spinal muscular atrophy types 2 and 3. Neurology 2012;79:1889-97.

[3] Finkel RS, Chiriboga CA, Vajsar J, Day JW, Montes J, De Vivo DC, et al. Treatment of infantile-onset spinal muscular atrophy with nusinersen: a phase 2, open-label, doseescalation study. Lancet 2016;388:3017-26.

[4] Mercuri E, Darras BT, Chiriboga CA, Day JW, Campbell C, Connolly AM, et al. Nusinersen versus Sham Control in Later-Onset Spinal Muscular Atrophy. N. Engl. J. Med. 2018;378:625-35.

[5] Pechmann A, Langer T, Wider S, Kirschner J. Single-center experience with intrathecal administration of Nusinersen in children with spinal muscular atrophy type 1. Eur. J. Paediatr. Neurol. 2018;22:122-7.

[6] Messina S, Pane M, Sansone V, Bruno C, Catteruccia M, Vita G, et al. Expanded access program with Nusinersen in SMA type I in Italy: Strengths and pitfalls of a successful experience. Neuromuscul. Disord. 2017;27:1084-6.

[7] Mercuri E, Finkel R, Montes J, Mazzone ES, Sormani MP, Main M, et al. Patterns of disease progression in type 2 and 3 SMA: Implications for clinical trials. Neuromuscul Disord 2016;26:126-31.

[8] Mercuri E, Lucibello S, Pera MC, Carnicella S, Coratti G, de Sanctis R, et al. Long-term progression in type II spinal muscular atrophy: A retrospective observational study. Neurology 2019;93:e1241-e7. 
[9] Berard C, Fermanian J, Payan C. Outcome measure for SMA II and III patients. Neuromuscul. Disord. 2008;18:593-4; author reply 4-5.

[10] Barois A, Mayer M, Desguerre I, Chabrol B, Berard C, Cuisset JM, et al. [Spinal muscular atrophy. A 4-year prospective, multicenter, longitudinal study (168 cases)]. Bull. Acad. Natl. Med. 2005;189:1181-98; discussion 98-9.

[11] Pane M, Staccioli S, Messina S, D'Amico A, Pelliccioni M, Mazzone ES, et al. Daily salbutamol in young patients with SMA type II. Neuromuscul. Disord. 2008;18:536-40.

[12] Main M, Kairon H, Mercuri E, Muntoni F. The Hammersmith functional motor scale for children with spinal muscular atrophy: a scale to test ability and monitor progress in children with limited ambulation. Eur. J. Paediatr. Neurol. 2003;7:155-9.

[13] O'Hagen JM, Glanzman AM, McDermott MP, Ryan PA, Flickinger J, Quigley J, et al. An expanded version of the Hammersmith Functional Motor Scale for SMA II and III patients. Neuromuscul. Disord. 2007;17:693-7.

[14] Mazzone E, De Sanctis R, Fanelli L, Bianco F, Main M, van den Hauwe M, et al. Hammersmith Functional Motor Scale and Motor Function Measure-20 in non ambulant SMA patients. Neuromuscul. Disord. 2014;24:347-52.

[15] Mazzone ES, Mayhew A, Montes J, Ramsey D, Fanelli L, Young SD, et al. Revised upper limb module for spinal muscular atrophy: Development of a new module. Muscle Nerve 2016.

[16] Pera MC, Coratti G, Mazzone ES, Montes J, Scoto M, De Sanctis R, et al. Revised upper limb module for spinal muscular atrophy: 12 month changes. Muscle Nerve 2019;59:426-30.

[17] Goemans N, Vanden Hauwe M, Signorovitch J, Swallow E, Song J, Collaborative Trajectory Analysis P. Individualized Prediction of Changes in 6-Minute Walk Distance for Patients with Duchenne Muscular Dystrophy. PLoS One 2016;11:e0164684. 
[18] Mercuri E, Signorovitch JE, Swallow E, Song J, Ward SJ, Group DMDI, et al. Categorizing natural history trajectories of ambulatory function measured by the 6-minute walk distance in patients with Duchenne muscular dystrophy. Neuromuscul. Disord. 2016;26:576-83. 


\section{Figure legends}

Figure 1: Baseline distribution by age in the 12-month cohort. Key to figure: Black line=Polynomial trendline on HFMSE total scores.

Figure 2: HFMSE changes distribution by age at 12 month $(A)$ and 24 month (B). HFMSE changes distribution by baseline at 12 month (C) and 24 month (D).

Figure 3: HFMSE score changes at 12 and 24 months (\%) by age and functional status. Black indicates a loss of more than 2 points, grey indicates stability \pm 2 points and white a gain of more than 2 points)

Figure 4 illustrates HFMSE score changes for the sitter cohort at 12 and 24 months (\%) by age and baseline HFMSE scores. Panel A: $<5$ years, Panel B: 5-13 years, Panel C: $>13$ years. Black indicates a loss of more than 2 points, grey indicates stability \pm 2 points and white a gain of more than 2 points) 
Tables

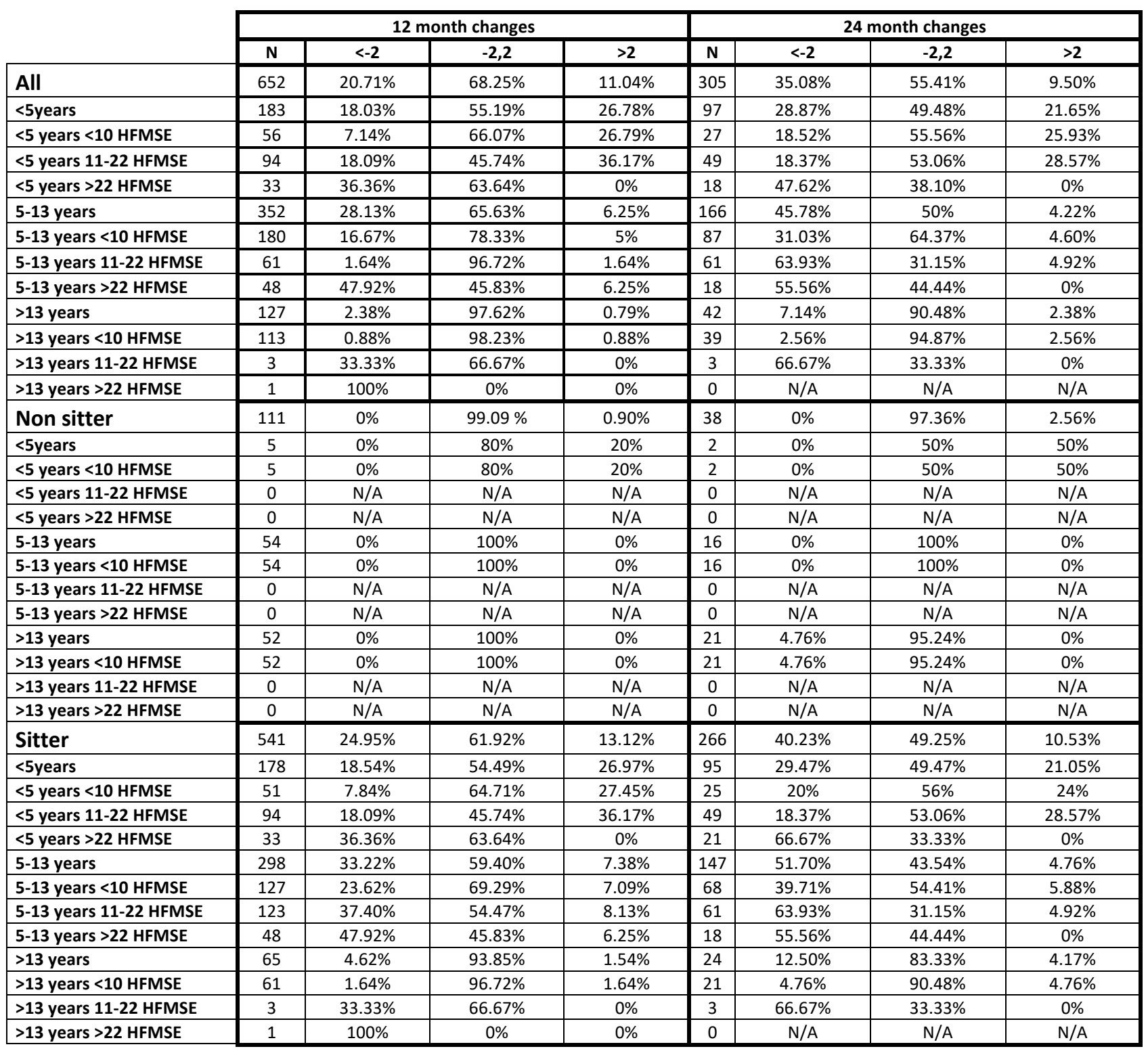

Table 1 illustrates score changes for cohort at 12 and 24 months (\%) subdivided by functional

status, age and baseline HFMSE scores. 


\section{ISMAC group:}

Jacqueline Montes, Amy Pasternak, Jessica Exposito Escudero, Elena Mazzone, Anna Mayhew, Allan M Glanzman, Sally Dunaway Young, Rachel Salazar, Roberto de Sanctis, Sara Carnicella, Evelin Milev, Marion Main, Marika Pane, Mariacristina Scoto, Laura Antonaci, Annalia Frongia, Julita Medina, Sonia Messina, Maria Sframeli, Gloria Ferrantini, Gianluca Vita, Marleen van den Hauwe, Emilio Albamonte, Chiara Marini-Bettolo, Francesca Bovis, Enrico Bertini, Francesca Salmin, Andres Nascimento Osorio, Richard Finkel. 